\title{
HRD1 sensitizes breast cancer cells to Tamoxifen by promoting S100A8 degradation
}

\author{
YanYang Wang ${ }^{1, *}$, AiBin Guo ${ }^{2, *}$, XiuBin Liang ${ }^{3}$, Min $\mathrm{Li}^{3}$, Ming Shi', Yan Li $^{4}$, Gareth \\ Jenkins ${ }^{5}$, XiaWen Lin ${ }^{1}$, XueFei $\mathrm{Wei}^{1}$, ZhiJun Jia ${ }^{1}$, XueFeng Feng ${ }^{1}$, DongMing Su ${ }^{4}$, \\ WanHua Guo ${ }^{1}$ \\ ${ }^{1}$ Department of Nuclear Medicine, The Affiliated Drum Tower Hospital of Nanjing University, Nanjing, China \\ ${ }^{2}$ Department of Geriatric Medicine, The Affiliated Drum Tower Hospital of Nanjing University, Nanjing, China \\ ${ }^{3}$ Department of Surgical Oncology, The First Affiliated Hospital With Nanjing Medical University, Nanjing, China \\ ${ }^{4}$ Center of Pathology and Clinical Laboratory, Sir Run Run Hospital, Nanjing Medical University, Nanjing, China \\ ${ }^{5}$ Institute of Advanced Materials, Nanjing University of Post and Telecommunication, Nanjing, China \\ *These authors contributed equally to this work
}

Correspondence to: DongMing Su, email: sudongming@njmu.edu.cn WanHua Guo, email: wanhuaguo@163.com

Keywords: Tamoxifen, HRD1, S100A8, chemotherapy resistance

Received: July 20, $2016 \quad$ Accepted: February 06, $2017 \quad$ Published: March 01, 2017

\section{ABSTRACT}

Estrogen receptor alpha positive (ER+) of breast cancer could develop resistance to antiestrogens including Tamoxifen. Our previous study showed that the E3 ubiquitin ligase HRD1 played an important role in anti-breast cancer. However, its role in chemotherapy resistance hasn't been reported. In this study, we found that HRD1 expression was downregulated in Tamoxifen-resistant breast cancer cell line MCF7/ Tam compared to the Tamoxifen sensitive cell line MCF7. Moreover, S100A8 is the direct target of HRD1 by proteome analysis. Our data showed that HRD1 decreased the protein level of S100A8 through ubiquitination while HRD1 was regulated by acetylation of histone. More importantly, HRD1 knockdown significantly increased the cell survival of MCF7 cells to the Tamoxifen treatment. HRD1 overexpression sensitized MCF7/Tam cells to the Tamoxifen treatment in vitro and in vivo. In conclusion, the decrease of HRD1 expression contributed to Tamoxifen resistance in breast cancer.

\section{INTRODUCTION}

The breast cancer is one of the most common cancers among the women in the whole world. Among the breast cancer, $70 \%$ are estrogen receptor alpha positive $(\mathrm{ER}+)$ breast cancer. Therefore, in the clinical, the major therapy is the Tamoxifen or other aromatase inhibitors in order to block estrogen receptor and inhibit the activation of downstream genes. However, about 50\% of ER+ breast tumors develop resistance to these drugs including Tamoxifen $[1,2]$. And thus contributes to the deaths of breast cancer patients. But, the understanding of the molecular mechanisms underlying the chemistry-drug resistance of breast cancer remains incomplete.

S100A8, a calcium-binding protein, is secreted primarily by granulocytes and monocytes, and is upregulated during the inflammatory response. It is reported that, $\mathrm{S} 100 \mathrm{~A} 8$ is associated with estrogen receptor loss in breast cancer [3]. Furthermore, S100A8 is involved in the chemistry-drug resistance in lots of kind of tumors. In leukemia cells, S100A8 promotes the autophagy so that to contribute to the drug resistance [4]. And in the breast cancer cells, the increased secretion of S100A8/A9 exacerbated the resistance of breast cancer to doxorubicin with cyclophosphamide [5]. More importantly, the CXCL1/2-S100A8/A9 loop is the cancer cell survival axis linking the chemotherapy resistance and metastasis in breast cancer and is hyperactivated by chemotherapy. Therefore, there is a possibility of clinically targeting this axis both to limit the dissemination of cancer cells and to diminish drug resistance [6].

In our recent study, we have found that HRD1, the E3 ubiquitin ligase, interacted with IGF receptor (IGF-1R) directly so that to decrease the protein level of IGF-1R through ubiquitination in both MDA-MB-231 cells and MCF7 cells. Moreover, HRD1 also took part in 
inhibiting the EMT in breast cancer cells [7]. Therefore, HRD1 may play as an anti-tumor role in breast cancer cells. However, whether HRD1 implicated the chemistrydrug resistance of breast cancer remains unknown. In this study, we examined the expression of HRD1 in MCF7/ Tam and MCF7 cells and investigated its function in degradation of S100A8, in order to better understand its role in chemistry-drug resistance of breast cancer and its potential implications for cancer therapy.

Evidence has shown that the acetylation status of histones implicated in the chemistry-drug resistance in tumors [8-12]. The acetylation of histone promoted gene expression by increasing the approachability of promoters to the transcription machinery $[13,14]$. We found that HRD1 was significantly downregulated in MCF7/Tam cells compared to MCF7 cells. We also found the histone deacetylase inhibitor, TSA, could upregulate HRD1 expression at a dose-dependent manner in MCF7 cells. We hypothesized that epigenetic modification might be involved in regulation of HRD1 expression in MCF7/Tam cells. Thus, we tested this hypothesis in both MCF7/Tam cells and MCF7 cells.

\section{RESULTS}

\section{HRD1 was downregulated in MCF7/Tam cells}

To better understand the biological mechanisms of chemoresistance, especially the resistance to hormone chemotherapy drugs in ER (+) breast cancer cells, we selected Tamoxifen sensitive and derived resistant breast cancer cell line pair (MCF7 and MCF7/Tam). To identify the differential sensitivity of the parental MCF7 and MCF7/Tam breast cancer cell lines to Tamoxifen, we first determined the PG (Percentage Growth) of Tamoxifen which is currently used for the treatment of ER $(+)$ breast cancer by SRB assay. As shown in Figure 1A, MCF7/ Tam cells showed resistance to Tamoxifen with higher concentrations $(10 \mu \mathrm{mol} / \mathrm{l})$ when the $\mathrm{PG}$ is $50 \%$ than MCF7 cells $(5 \mu \mathrm{mol} / 1)$.

We then examined the expression of HRD1 in both protein and mRNA levels in MCF7 and MCF7/Tam cells. As shown in Figure $1 \mathrm{~B}$ and $1 \mathrm{C}$, the expression of HRD1 in MCF7/Tam cells significantly decreased in both protein and mRNA level compared to the MCF7 cells. These data suggested that, the downregulation of HRD1 may contribute to the resistance to Tamoxifen treatment.

\section{HRD1 directly interacted with S100A8}

In order to further explore the probability of chemoresistance, we overexpressed Vector or HRD1 in MCF7 cells and then did IP assay. As shown in Supplementary Figure 1A, 23 proteins were identified with obviously changes compared between overexpression of Vector and HRD1 (ESM Table 1). Among these identified proteins, $\mathrm{S} 100 \mathrm{~A} 8$ is related to chemoresistance of breast cancers. Supplementary Figure 1B shows the relative abundance of each mass fragment in both two samples of S100A8.

To verify the results from proteome analysis, we then did the Co-IP. As shown in Figure 2A, HRD1 interacted with S100A8 in MCF7 cells by Co-IP. Furthermore, the results of immunofluorescence show that, HRD1 and S100A8 co-located in the cytoplasm of the breast cancer cells (Figure 2B).

\section{HRD1 promoted the degradation of S100A8 through ubiquitination}

In MCF7 cells, overexpression of HRD1 inhibited S100A8 protein level at a dose-dependent manner (Figure 3A). For HRD1 is an E3 ubiquitin ligase, we further explored the potential mechanisms related to the decrease of HRD1 on S100A8. We treated MCF7 cells with cycloheximide (CHX), an inhibitor of protein synthesis, resulted in promotion of S100A8 degradation by overexpressing HRD1 (Supplementary Figure 2A). However, this degradation was suppresses by HRD1 knockdown (Figure 3C).

Moreover, an elevation in ubiquitinated S100A8 was found. As shown in Figure 3D, when inhibited the degradation of S100A8 by MG132, overexpression of HRD1 further increased the ubiquitination of S100A8. Therefore, these results indicated that HRD1 served as an E3 ubiquitin ligase so that to promote the ubiquitination of S100A8 to degrade by the proteasome.

\section{HRD1 was upregulated by acetylation of histone}

To determine whether the expression of HRD1 is regulated by methylation of DNA or by acetylation of histone, MCF7 cells were treated with different concentration of 5'-AZA, a DNA methyltransferases inhibitor and TSA, a histone deacetylases inhibitor for about $72 \mathrm{~h}$, followed by real-time PCR assay and Western blotting. The results showed that the treatment of 5'-AZA didn't change the expression of HRD1 at both mRNA and protein level. However, the treatment of TSA upregulated the expression of HRD1 at both mRNA and protein level at a dose-dependent manner compared to the control cells (Figure 4A, 4B). These results indicated that HRD1 was regulated by acetylation of histone. However, we also tested the expression of S100A8 at both mRNA and protein level by the treatment of TSA as well as $5^{\prime}$-AZA. The result showed hat the treatment of TSA can increase the mRNA expression of S100A8 at a dose-dependent manner while 5'-AZA cannot. But, the protein expression of S100A8 didn't change. It may due to both the increased mRNA expression of S100A8 and the increased protein expression of HRD1 (Supplementary Figure 3). 
Table 1: The results of proteome analysis

\begin{tabular}{lccccc}
\hline \multicolumn{1}{c}{ Gene Name } & Peptides & Unique Peptides & Mol.Weight [kDa] & iBAQ (Ctr > 0) & iBAQ (Hrd1/Ctr) \\
\hline PFKP & 22 & 20 & 85.595 & 6578.3 & 1.78 \\
PFKL & 8 & 6 & 85.018 & 113.61 & 4.63 \\
ACTG1 & 6 & 6 & 41.792 & 311.25 & 2.33 \\
RPL17 & 2 & 2 & 19.586 & 69.356 & 1.74 \\
RPS14 & 2 & 2 & 16.273 & 133.86 & 1.23 \\
PKM & 2 & 2 & 8.9512 & 203.02 & 3.53 \\
PRDX1 & 2 & 2 & 18.976 & 220.91 & 2.16 \\
S100A8 & 1 & 1 & 10.834 & 130.31 & 1.14 \\
ODF1 & 1 & 1 & 28.366 & 478.03 & 1.18 \\
NME1 & 1 & 1 & 6.5266 & 527.21 & 1.18 \\
DUSP28 & 1 & 1 & 18.324 & 1003 & 1.74 \\
LRMP & 1 & 1 & 38.995 & 11837 & 1.12 \\
LAMB1 & 1 & 1 & 10.082 & 27795 & 1.45 \\
ANKFY1 & 6 & 6 & 128.4 & 149.6 & 0.71 \\
EEF1A1P5 & 4 & 1 & 50.184 & 960.81 & 0.60 \\
ATP5O & 4 & 4 & 23.277 & 1133.8 & 0.60 \\
RPS18 & 3 & 3 & 17.718 & 270.06 & 0.42 \\
CFL1 & 2 & 2 & 10.181 & 379.28 & 0.80 \\
RPS25 & 2 & 2 & 13.742 & 635.14 & 0.34 \\
DCD & 2 & 2 & 11.284 & 723.04 & 0.31 \\
RPS20 & 2 & 2 & 13.373 & 962.15 & 0.51 \\
HST1H4A & 1 & 1 & 11.367 & 574.18 & 0.69 \\
MPI & 1 & 1 & 19.385 & 873.03 & 0.71 \\
\hline
\end{tabular}

Next, we did ChIP-qPCR assay to test whether the downregulation of HRD1 in MCF7/Tam cells is due to the downregulation of acetylation of histone in the HRD1 promotor region. The results showed that acetylhistone $\mathrm{H} 3$ and acetyl-histone $\mathrm{H} 4$ could bind to the HRD1 promotor region (Figure 4C). Moreover, we found that the acetylation of histone $\mathrm{H} 3$ and histone $\mathrm{H} 4$ in the promoter region in MCF7/Tam cells were downregulated compared to MCF7 cells (Figure 4D). These results suggested that HRD1 was regulated by acetylation of $\mathrm{H} 3$ and $\mathrm{H} 4$ histone.

\section{HRD1 was involved in breast cancer resistance by directly targeting S100A8}

To further investigate the association of HRD1 expression with breast cancer chemoresistance, we constructed S100A8 plasmid and chose the SiRNA of S100A8 (Figure 5A, 5B). Because of the expression of HRD1 in MCF7 cells was higher than MCF7/Tam cells, we transfected Si-NC, Si-HRD1 and/or Si-S100A8 in MCF7 cells while transfected vector, HRD1 and/or S100A8 in MCF7/Tam cells, followed by treatment of $5 \mu \mathrm{mol} / 1$ Tamoxifen in MCF7 cells and $10 \mu \mathrm{mol} / 1$ Tamoxifen in MCF7/Tam cells. The inhibition of cell proliferation by the Tamoxifen was obviously decreased by knockdown of HRD1 in MCF7 cells, which was reversed by S100A8 deletion (Figure 5C). Meanwhile, the inhibition of cell proliferation by the Tamoxifen was significantly increased by overexpression of HRD1 in MCF7/Tam cells, which was reversed by overexpression of S100A8 (Figure 5D). Moreover, the results of flow cytometry showed that knockdown of HRD1 in MCF7 cells reduced the apoptosis rate of cells with the treatment of $5 \mu \mathrm{mol} / 1$ Tamoxifen while overexpression of HRD1 in MCF7/Tam cells increased the apoptosis rate of cells with the treatment of $10 \mu \mathrm{mol} / 1$ Tamoxifen (Figureure 5E-5G, Supplementary Figure 4A). These results indicated that overexpression of HRD1 could sensitize MCF7/Tam cells to the treatment of Tamoxifen while knockdown of HRD1 can be resistant MCF7 cells to Tamoxifen.

\section{Overexpression of HRD1 increased the sensitivity of drug-resistant breast tumors to tamoxifen treatment in vivo}

We explored the in vivo effects of HRD1 on the breast cancer chemoresistance by injecting MCF7/Tam cells stable overexpressing HRD1 or the corresponding controls into nude mice, respectively (Supplementary Figure 5). As expected, overexpresssion of HRD1 significantly 

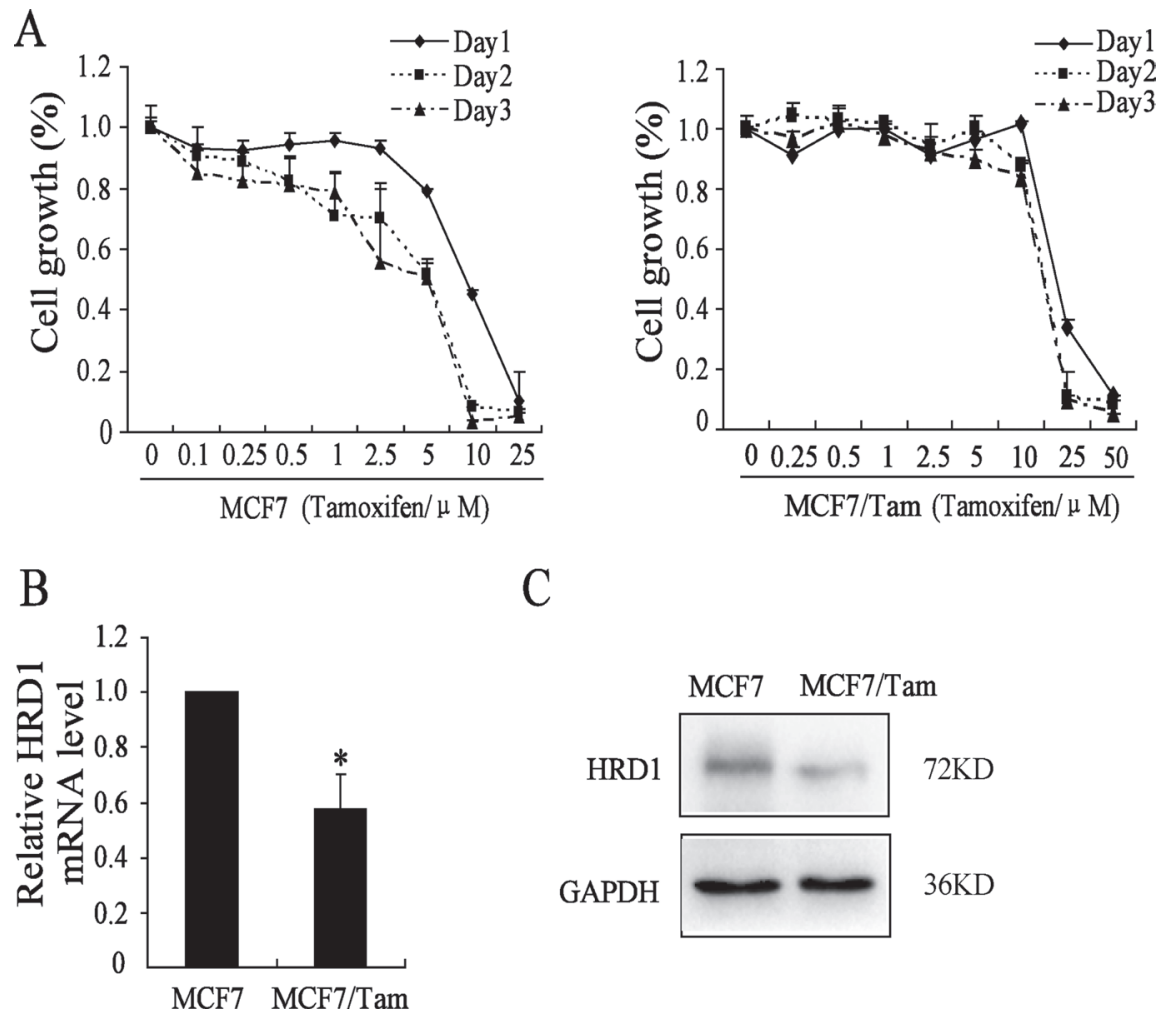

$\mathrm{C}$

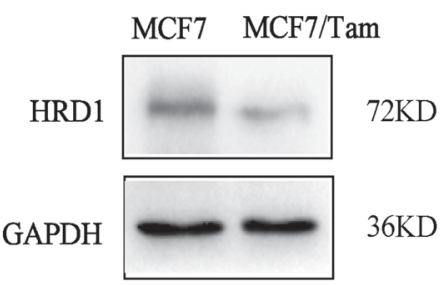

Figure 1: HRD1 is downregulated in MCF7/Tam cells. (A) Cells were treated with various concentrations of Tamoxifen for about $24 \mathrm{~h} / 48 \mathrm{~h} / 72 \mathrm{~h}$. Survived cells were measured by SRB assay. SRB assay shows that MCF7/Tam cells are much more resistant to Tamoxifen than MCF-7 cells. (B) Western blotting analysis showing the protein expression of HRD1 in MCF7 and Tam-R MCF7 cells. The protein level of HRD1 in MCF7 cells are much more than MCF7/Tam cell. GAPDH was used as an internal loading control. (C) Realtime PCR indicates a significant down-regulation of HRD1 in mRNA level in MCF7/Tam cells compared with MCF7 cells. All graphs show means \pm S.D. of three independent experiments, ${ }^{*} P<0.05$, compared to MCF7.

A
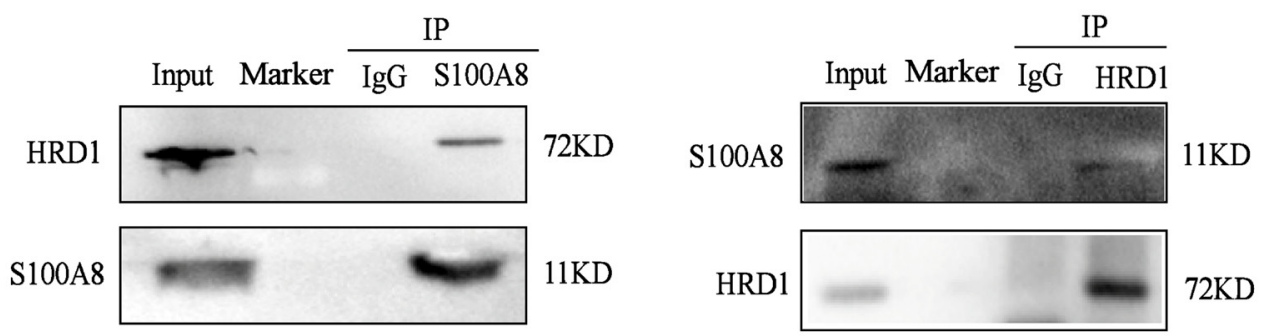

B

HRD1

S100A8
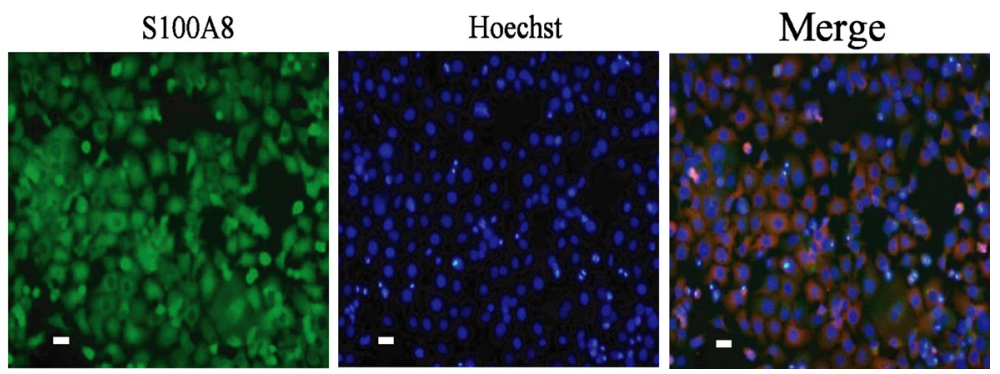

Figure 2: HRD1 directly interacted with S100A8. (A) MCF-7 cells were pretreated with MG132 (10 $\mu$ mol/l) for $6 \mathrm{~h}$ and endogenous protein-protein interactions between HRD1 and S100A8 were determined by immunoprecipitation (IP) with HRD1 or S100A8 antibodies, followed by immunoblotting. IgG was used as a negative control for IP. (B) Inmmunofluorescence to certificate HRD1 and S100A8 can co-locate in the cytoplasm in MCF7 cells (Scale bar $=20 \mu \mathrm{m})$. 

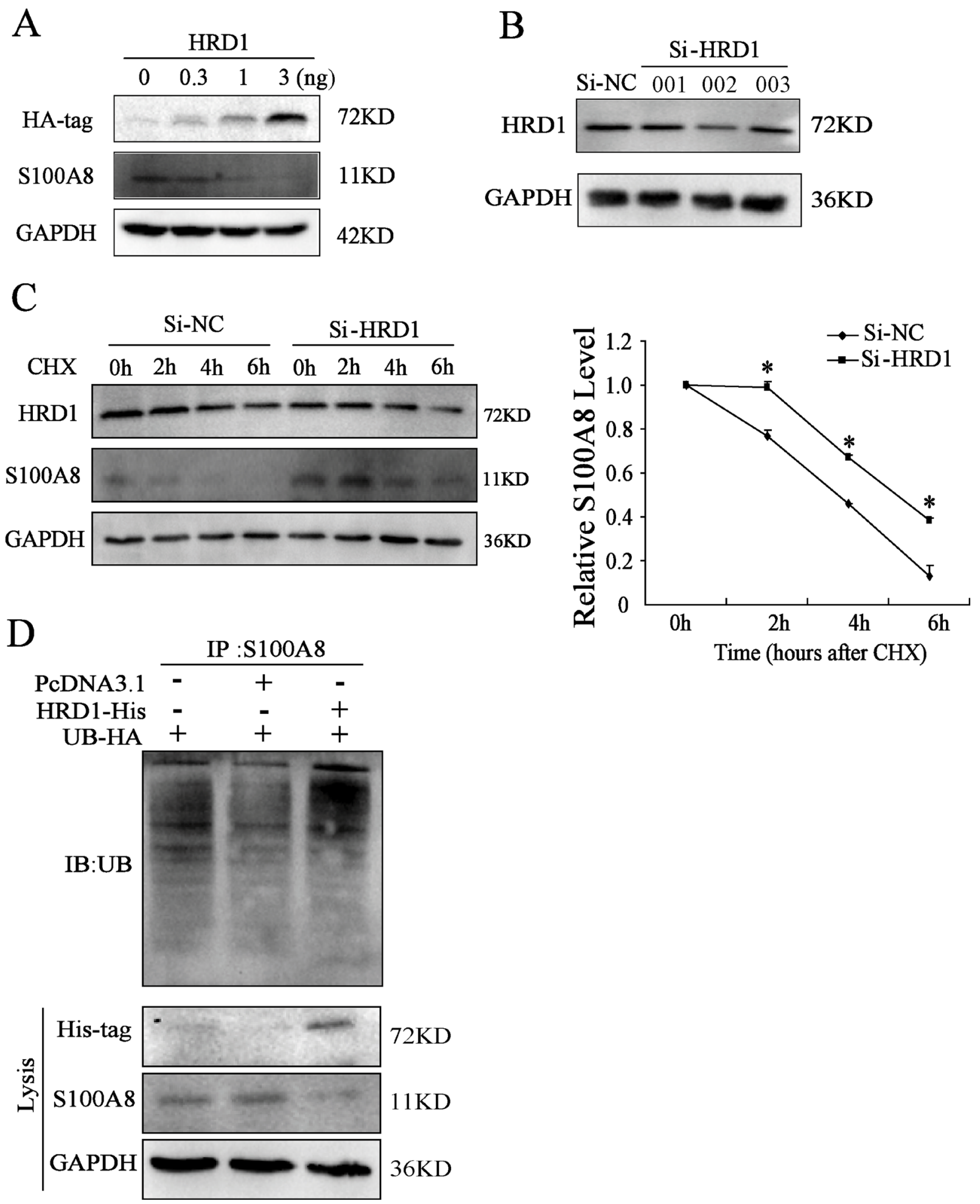

Figure 3: HRD1 promotes the degradation of S100A8 through ubiquitination. (A) The protein level of HRD1, S100A8 through Western blotting analysis in MCF7 cells with overexpression of different dose of HRD1. (B) The protein level of HRD1 in MCF7 cells by knockdown HRD1 through Western blotting analysis. (C) MCF7 cells were transfected with si-HRD1 and the control for 48 h, followed by exposure to cycloheximide (CHX 50ng/ml) for 0, 2, 4, $6 \mathrm{~h}$. The protein of S100A8 and HRD1 in whole cell lysates was measured. (D) Ubiquitination of S100A8 was induced by HRD1. HA-ubiquitin was co-expressed in MCF7 cells with His-HRD1 or Vector control with treatment of MG132 (10 $\mu \mathrm{mol} / \mathrm{l})$ for $4 \mathrm{~h}$. Ubiquitinated S100A8 was immunoprecipitated using S100A8 antibody and further detected with UB antibody. The endogenous S100A8 and His-HRD1 in the whole cell lysates were examined by S100A8 and His-tag antibodies. ${ }^{*} P<0.05$, compared to Si-NC. 
increased the sensitivity of MCF7/Tam cells to Tamoxifen. As indicated in Figure 6A, the growth of xenograft tumors was much slower in the group transfected with EGFPtagged HRD1 lentivirus than in the group transfected with EGFP lentivirus. However, the body weight did not exhibit obvious difference between the two groups (Figure 6B).

\section{DISCUSSION}

In nowadays, breast cancer becomes one of the most common cancers among the women in the worldwide. However, about $50 \%$ ER+ breast cancer will be onset of resistance as well as recurrence after the treatment

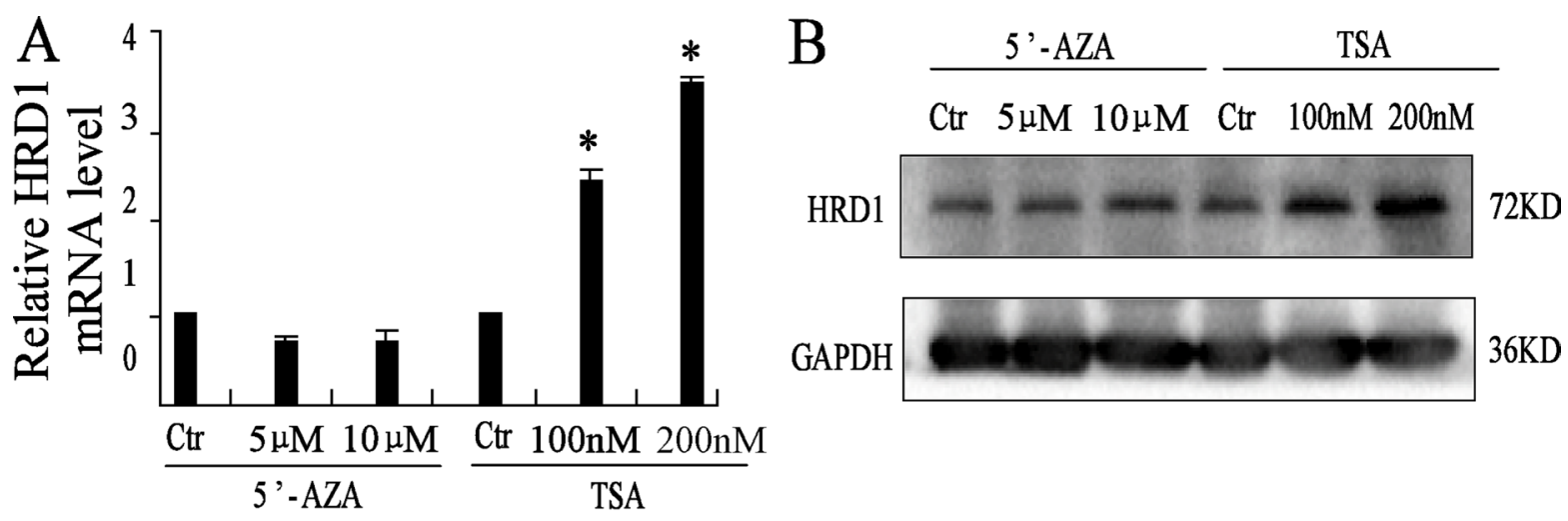

$\mathrm{C}$

MCF7 HRD1 Promotor
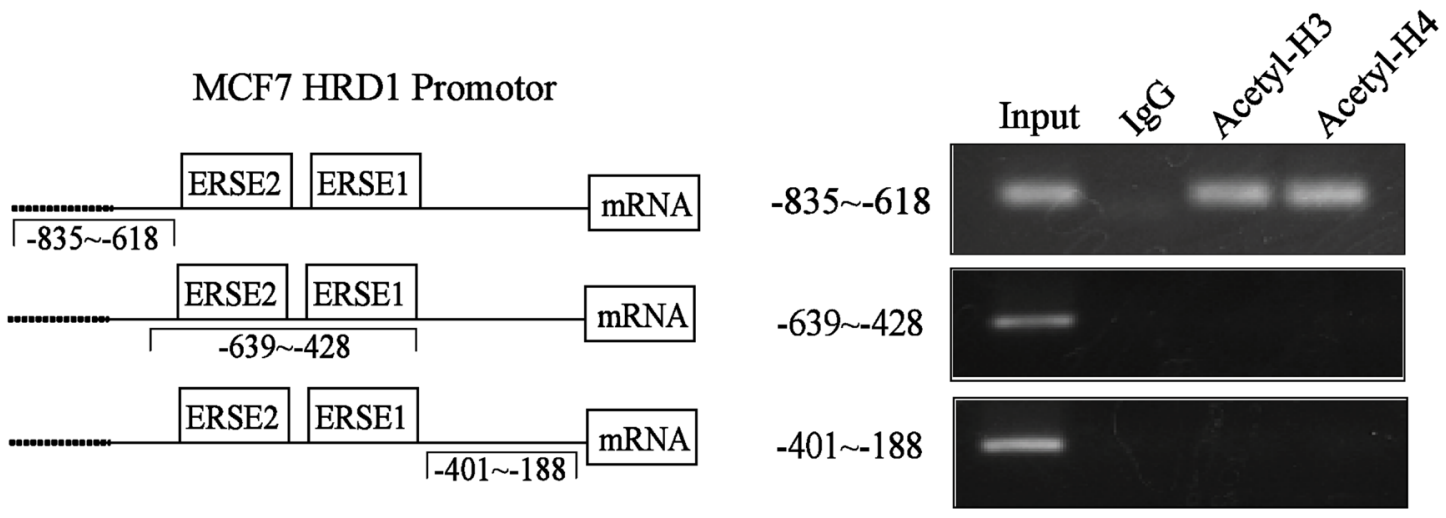

$\mathrm{D}$
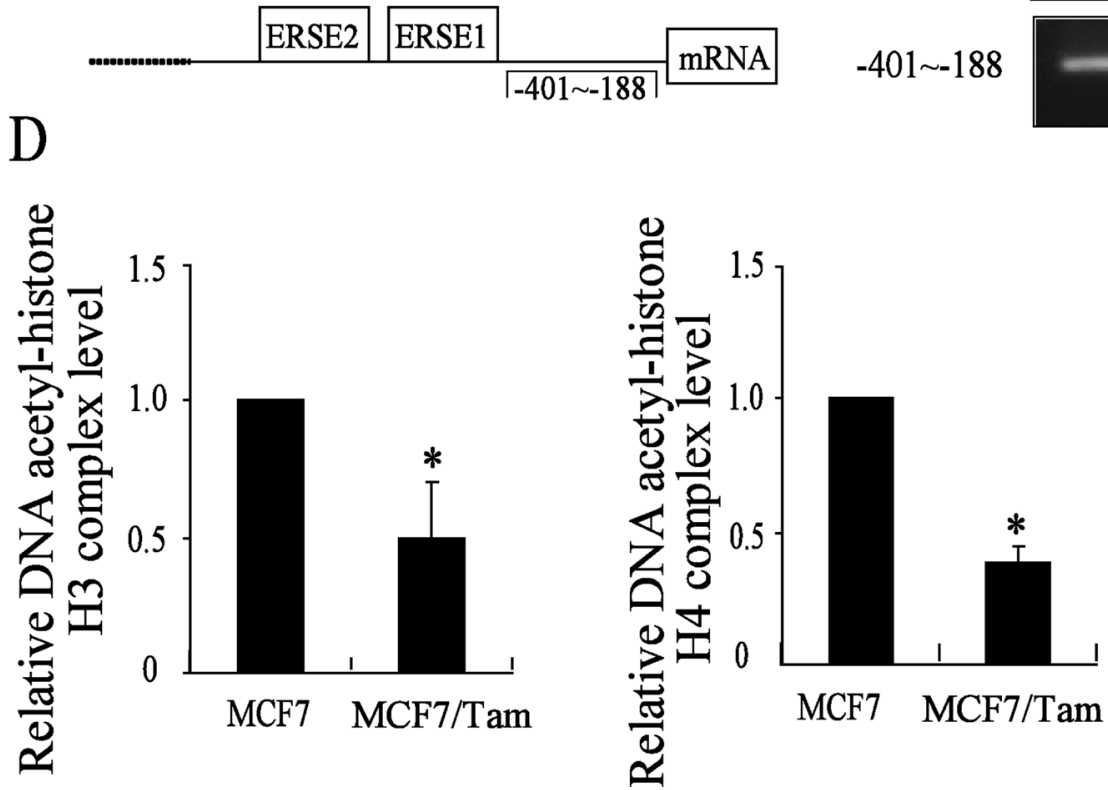

Figure 4: HRD1 is upregulated by acetylation of histone. (A) Different concentration of 5'AZA and TSA used in the MCF7 cells for $72 \mathrm{~h}$, expression of HRD1 protein level determined by Western blotting. (B) Different concentration of 5'AZA and TSA used in the MCF7 cells for $72 \mathrm{~h}$, expression of HRD1 mRNA level determined by qRT-PCR. (C) The HRD1 promotor region mode pattern on the left. On the right, CHIP assay did with anti acetyl-histone H3 and H4 and negative control anti-IgG (Rabbit). A agarose gel (1\%) of PCR products amplified with primers to the region of HRD1 promotor respectively (-835 -618, $-639 \sim-428,-401 \sim-188)$. (D) qRT-PCR tests the Dna-Acetyl-histone H3 and H4 binding levels in both MCF7 cells and MCF7/Tam cells. All graphs show means \pm S.D. of three independent experiments, $* P<0.05$, compared to MCF7. 
A
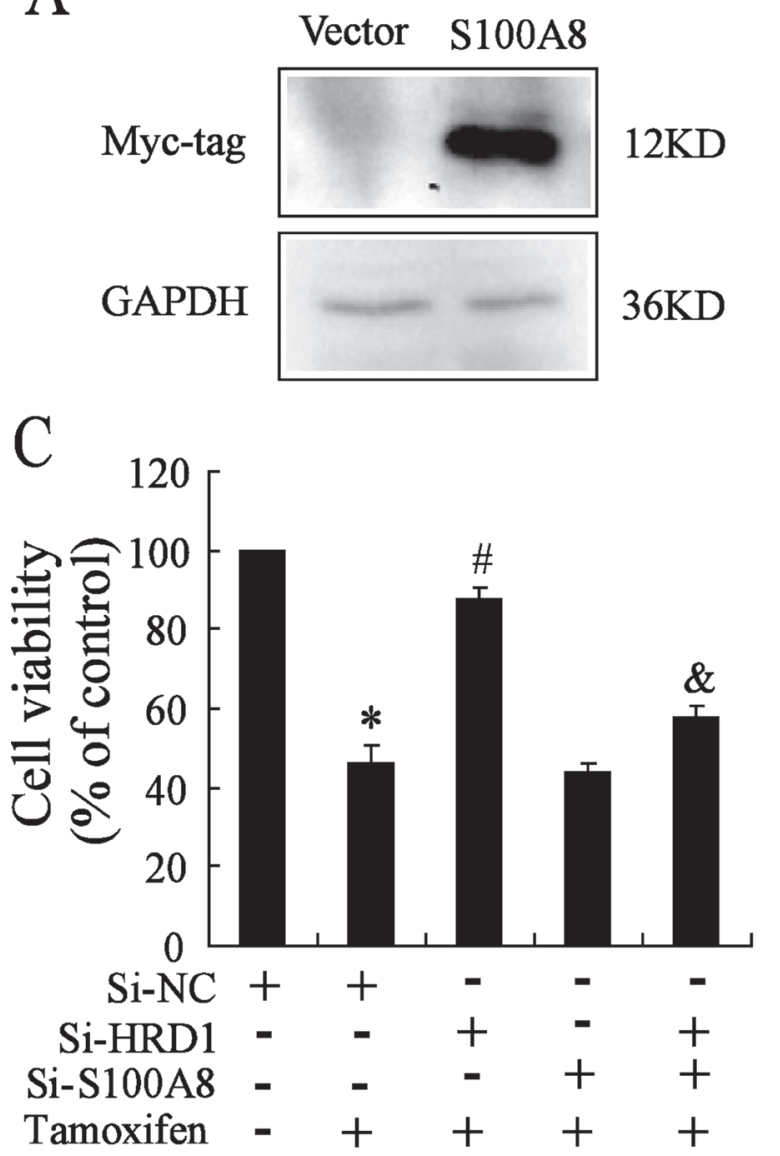

$\mathrm{E}$

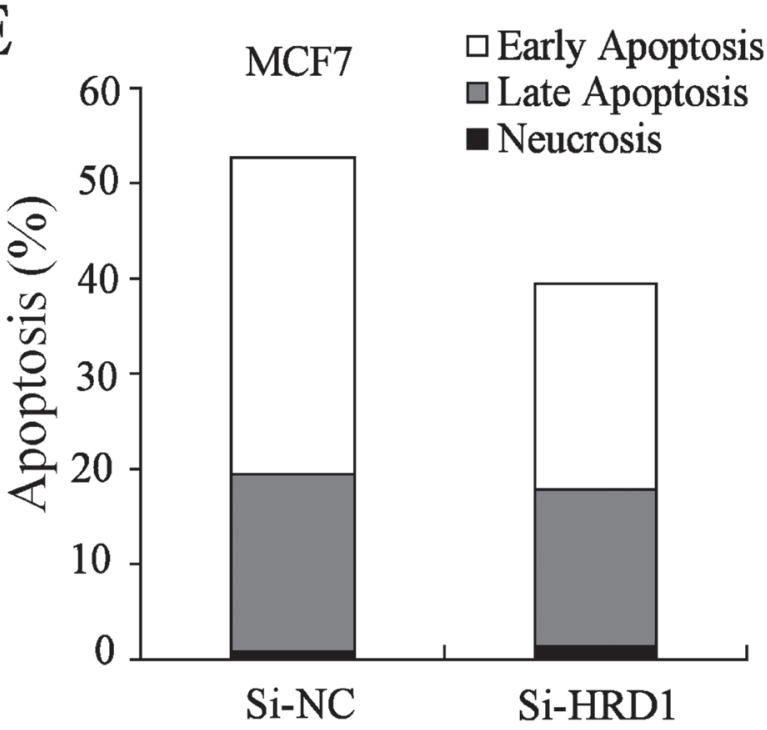

$\mathrm{B}$

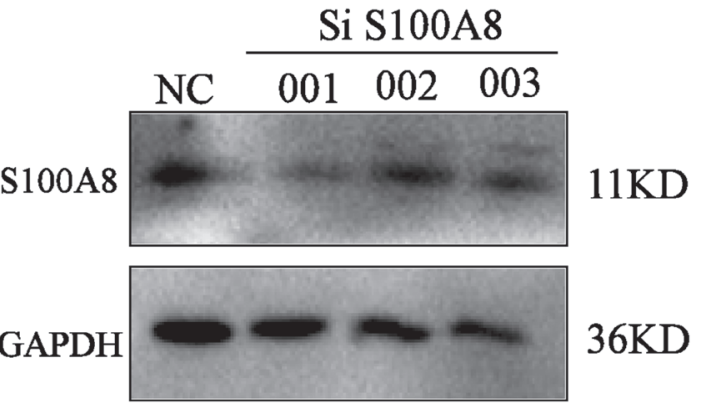

D

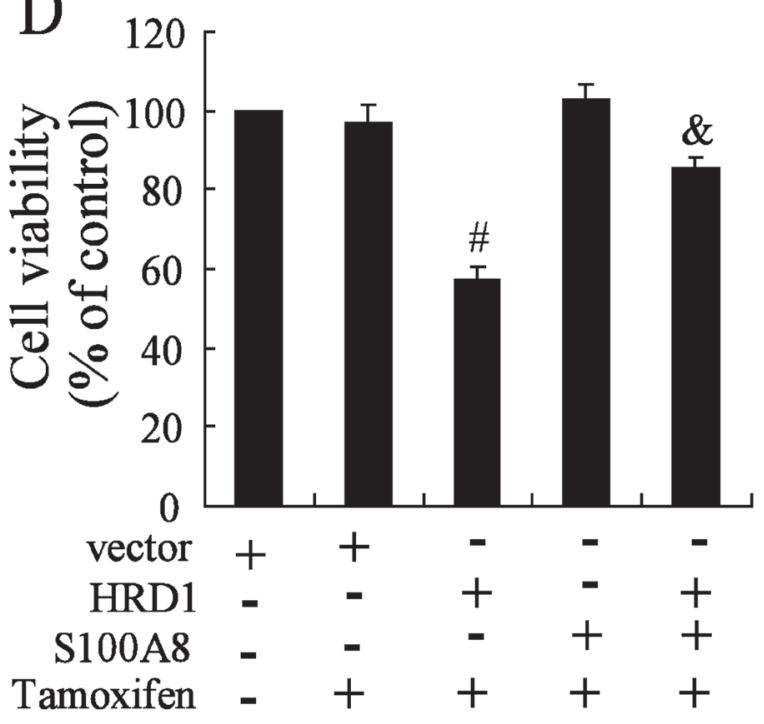

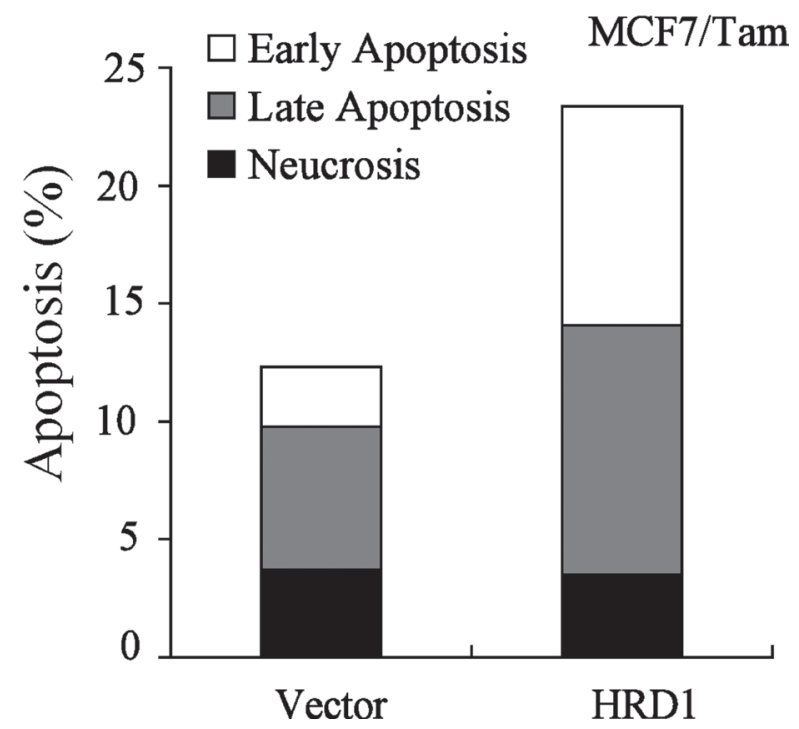

Figure 5: HRD1 can sensitize MCF-7 and MCF7/Tam cells to Tamoxifen. (A) S100A8 plasmid is constructed and tested by Western blotting. (B) Knockdown of S100A8 in MCF7 cells and tested by Western Blot. (C) MCF7 cells were transfected SiNC, SiHRD1, SiS100A8 and co-transfected SiHRD1 and SiS100A8 respectively, followed by treatment of $5 \mu \mathrm{M}$ Tamoxifen. After 48 h, SRB assay played. (D) MCF7/Tam cells were transfected Vector, HRD1, S100A8 and co-transfected HRD1 and S100A8 respectively, followed by treatment of $10 \mu \mathrm{mol} / 1$ Tamoxifen. After $48 \mathrm{~h}$, SRB assay played. (E) MCF7 cells were transfected SiNC and SiHRD1 respectively for $24 \mathrm{~h}$ followed by treatment of $5 \mu \mathrm{mol} / 1$ Tamoxifen. After $48 \mathrm{~h}$, the flow cytometry played. (F) MCF7/Tam cells were transfected Vector and HRD1 plasmid respectively for $24 \mathrm{~h}$ followed by treatment of $10 \mu \mathrm{mol} / 1$ Tamoxifen. After $48 \mathrm{~h}$, the flow cytometry was performed. All graphs show means \pm S.D. of three independent experiments. ${ }^{* *} P<0.05$, compare to $\mathrm{Si}-\mathrm{NC}$ or vector; ${ }^{*} P<0.05$, compare to Si-NC or vector + Tamoxifen; ${ }^{\&} P<0.05$, compare to Si-HRD1 or HRD1 + Tamoxifen. 
of Tamoxifen and other aromatase inhibitors [1, 2]. Therefore, the breast cancer is still the major killer for the women in the whole world. In our study, we have found the E3 ubiquitin ligase of HRD1 was involved in the Tamoxifen resistance of breast cancer and could be the new target for the treatment of ER + breast cancer in the clinical.

Acetylation status of histones has been shown to be involved in the drug resistance in breast cancer. Histone decetylase 4 (HDAC4) could mediate the deacetylation of SMAD family 4 so that induce the 5-fluorouracil resistance in both ER+ and ER- breast cancer cells [15]. Moreover, in $\mathrm{ER}+$ breast cancer cells, the resistance to fulvestrant was due to the modulated expression of GPER and CDK6 in which the deacetylase was implicated [16]. In this study, we found that the histone deacetylase inhibitor (TSA) could upregulate HRD1 expression in breast cancer cells. However, the specific inhibitor of DNA methylation (5-Aza-CdR) had no effect on HRD1 expression. These results indicated that HRD1 is regulated by acetylation of histone in breast cancer cells. Further studies using ChIP analysis confirmed that $\mathrm{H} 4$ and $\mathrm{H} 3$ histone bound to the promoter region of HRD1 ( -835 to -618$)$, which is adjoined to two ER stress response elements (ERSE1 and ERSE2) [17-18]. Further investigation of correlation between acetylation of histone and ERSE will be benefit to understand how HRD1-mediated drug resistance in breast cancer in future.

It is also important to recognize that acetylation of histone $\mathrm{H} 3$ and $\mathrm{H} 4$ in HRD1 promoter was significantly decreased in MCF7/Tam cells. These results clearly demonstrate that the lower acetylation of histone resulted in the lower expression of HRD1 in MCF7/Tam cells

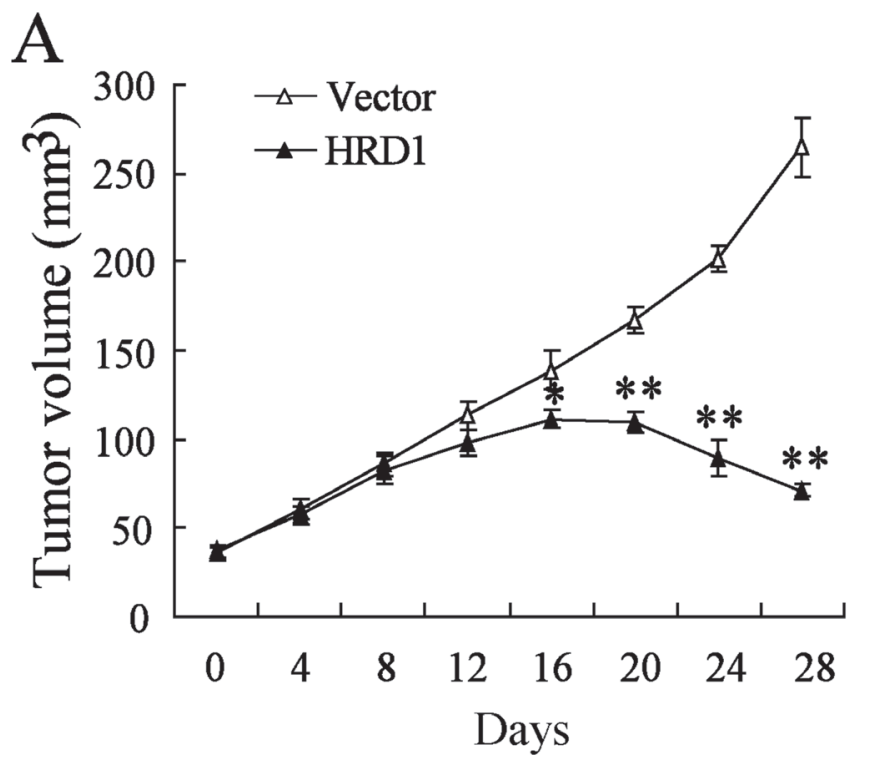

compared to MCF7 cell. Meanwhile, overexpression of HRD1 in MCF7/Tam cells increased the response to Tamoxifen, while inhibition of HRD1 in MCF7 cells decreased the response to Tamoxifen. More importantly, HRD1 overexpression increased the sensitivity of drugresistant breast tumors to Tamoxifen treatment in animal model. These results indicated that HRD1 mediated chemotherapy resistance of breast cancer.

We identified for the first time S100A8 as the downstream target of HRD1 in mediating its drug-resistant effects on breast cancer cells. S100A8 plays an important role in chemotherapy resistance and metastasis of breast cancer $[3,6]$. In the present study, we found that HRD1 downregulated S100A8 expression. Furthermore, we demonstrated that HRD1 could interact with S100A8 acting as a ubiquitin E3 ligase that targeted S100A8 for degradation through proteasomal degradation pathway. In MCF7 cells, S100A8 deletion reversed the effect of knockdown of HRD1 on Tamoxifen treatment. In MCF7/ Tam cells, S100A8 overexpression reversed the effect of upregulation of HRD1 on Tamoxifen treatment. Therefore, HRD1 mediated breast cancer resistance by directly inhibiting S100A8 expression.

In conclusion, our findings indicated that HRD1 expression was negatively correlated with the Tamoxifen resistance of ER+ breast cancers. The lower acetylation of histone was responsible for the downregulation of HRD1 in breast cancer cells. Overexpression of HRD1 increased the response of breast cancer to Tamoxifen by inhibiting S100A8 expression. Based on our findings, we proposed that restoration of HRD1 expression may be an improved strategy for endocrine therapy for human ER+ breast cancers.

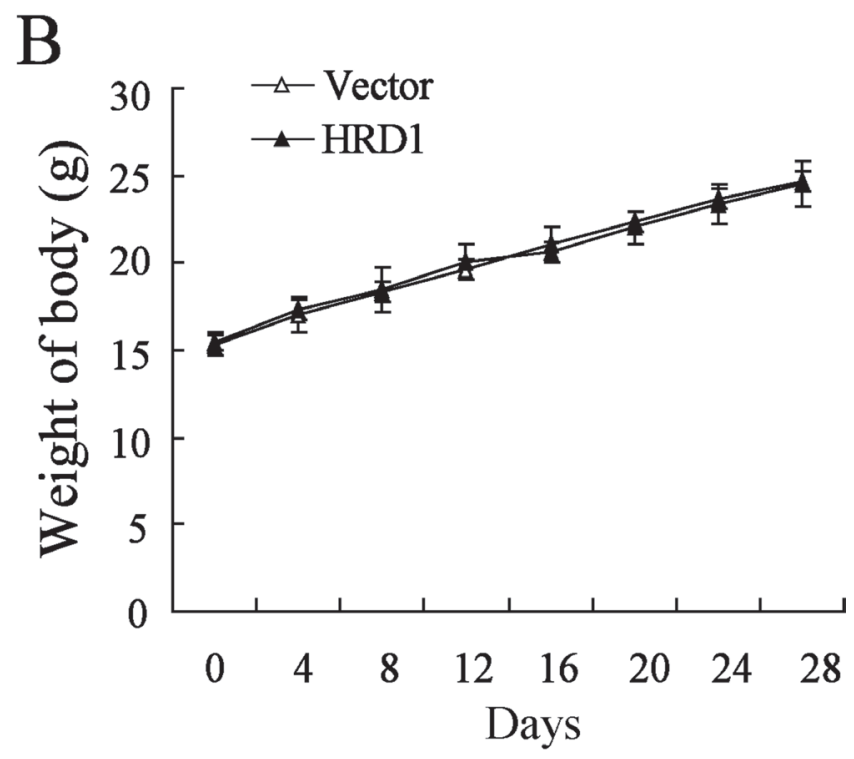

Figure 6: Overexpression of HRD1 increased the sensitivity of drug-resistant breast tumors to Tamoxifen treatment in vivo. MCF7/Tam cells stably overexpressing of HRD1 and the corresponding controls were injected into the right flank and left flank of nude mice, respectively. Tumor volume (A) and body weights of mice with tumors (B) were measured. ${ }^{*} P<0.05$, compared to vector. 


\section{MATERIALS AND METHODS}

\section{Cell lines and culture}

The Tamoxifen resistance human breast cancer MCF7/Tam cells and Tamoxifen sensitive human breast cancer MCF7 cells were gifts from Doc. Chen (Nanjing Southeast University). The MCF7/Tam cells and MCF7 cells were cultured in RPMI-1640 (Invitrogen, Carlsbad, CA) supplemented with $10 \%$ Fetal Bovine Serum (FBS) and were added with the indicated $1 \mu \mathrm{mol} / 1$ Tamoxifen for resistance maintenance. All cells were cultured at $37^{\circ} \mathrm{C}$ in a humidified atmosphere containing 95\% air and 5\% CO2 [19].

\section{Cell transfection}

MCF7 and MCF7/Tam cells were transfected with different amount of HRD1 and S100A8 or the vector pCMV5-myc using Lipofectamine 2000, according to the manufacturer's instruction. After transfection for $24 \mathrm{~h}$, cells were then treated with different concentration of Tamoxifen, and then cells were used for Western blot and qRT-PCR.

\section{RNAi plasmid and plasmid construction}

HRD1 and S100A8 expression was silenced utilizing specific small interfering RNA (HRD1-siRNA, S100A8siRNA and Si-NC) purchased from Genechem (Shanghai, China). HRD1-siRNA and Si-NC were the same as described in our previous study [7]. And the sequence of S100A8siRNA wAS on ESM Table 1. The S100A8 expression plasmid was constructed by inserting the full-length coding region sequences into pCMV5-myc vector between NdeI and BamHI. The primer sequence of the full-length coding region of S100A8 is: 5'-GGAATTCCATATGATGTTGAC CGAGCTGGAG-3' (Forward), 5'-CGGGATCCCTACTC TTTGTGGCTTTCTTC-3' (Reverse). The HRD1 expression plasmid was described previously [7]. All constructions used here were sequenced and confirmed to be correct.

\section{Co-immunoprecipitation (Co-IP) and proteome analysis}

Co-immunoprecipitation (Co-IP) was performed according to our previous methods [7]. And after using SDS-PAGE to test the IP samples staining by CBB (Coomassie Brilliant Blue), we then cleaved the two whole sample lanes to submit the cleaved lanes to Analysis and Test Centre of Nanjing Medical University to do the proteome analysis.

\section{SRB assay for cell survival}

MCF7 and MCF7/Tam cells were seeded onto 96-well plates at a density of 1,000 cells/well. After culture for $24 \mathrm{~h}$, cells were transfected with siRNA or overexpression plasmid $24 \mathrm{~h}$. Then, MCF7 cells were treated with $5 \mu \mathrm{mol} / 1$ Tamoxifen and MCF7/Tam cells were treated with $10 \mu \mathrm{mol} / 1$ Tamoxifen for $48 \mathrm{~h}$. Next, cells were treated with $10 \% \mathrm{TCA}(\mathrm{w} / \mathrm{v})$ for $12 \mathrm{~h}$. And then, $0.4 \%$ SRB (Sigma, USA) was added for about $30 \mathrm{~min}$. With $10 \mu \mathrm{mol} / 1 \mathrm{PH}=10.5$ Tris-base, the absorbance at $540 \mathrm{~nm}$ was measured using a multi-mode reader (LD942, Beijing, China). Besides, MCF7 and MCF7/Tam cells were seeded onto 96-well plates at a density of 1,000 cells/well. After culture for $24 \mathrm{~h}$, cells were treated with serial dilutions of Tamoxifen for $48 \mathrm{~h}$, followed by SRB Assay. The IC50 (50\% inhibitory concentration) value was calculated by normal probability transforms according to the relationship of drug concentration and inhibition rate.

\section{Real-time PCR assay}

Real-time PCR assay was performed to detect the relative expression of mRNA. Briefly, total RNA was isolated from MCF7 and MCF7/Tam cells using Trizol reagents (Invitrogen, CA, USA) according to the manufacture's protocol. RNA was reverse transcribed into complementary DNA using ReverTra ACE (Toyobo, Shanhai, China). Realtime PCR assay was performed using SYBR Premix Ex

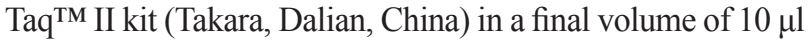
mixture containing $1 \mu \mathrm{l} \mathrm{cDNA}, 0.5 \mu \mathrm{l}$ of each primer, and $5 \mu \mathrm{l}$ SYBRGreen. Relative mRNA expression was normalized to GAPDH expression. Sequences of the primers used were available in ESM Table 1.

\section{Western blotting}

MCF7 and MCF7/Tam cells were washed with ice-cold PBS for 2 times, and then were added with RIPA lysis buffer. Protein in each sample was analyzed by western blotting as previously reported [7]. The dilution of each antibodies is: anti-S100A8 (1:500) (Proteintech, Chicago, USA), anti-HRD1 (1:1000) (Abcam, Cambridge, UK), anti-GAPDH (1:4000) (Abcam, Cambridge, UK), anti-HA-tag (1:1000) (Proteintech, Chicago, USA), anti-His-tag (1:1000) (Santz Cruz, CA, USA), anti-Myc-tag (1:1000) (Proteintech, Chicago, USA).

\section{Flow cytometry}

Flow cytometry was performed on a Guava easyCyte HT System Flow Cytometer (Millipore) using Annexin V-FITC Apoptosis Detection Kit (Vazyme, Nanjing, China) for analysis of early and late apoptotic cells according to the manufacturer's instructions (Vazyme).

\section{Chromatin immunoprecipitation (ChIP) assay and ChIP-qPCR assay}

ChIP assays were performed using a commercially available ChIP Assay Kit (Millipore) according to our previous methods [7]. Sequences of the primers used were available in ESM Table 2. 


\begin{tabular}{|c|c|c|}
\hline Construct & & Sequences \\
\hline \multicolumn{3}{|c|}{ Primers for real-time PCR } \\
\hline \multirow[t]{2}{*}{ HRD1 } & Sense & 5'-AACCCCTGGGACAACAAG-3' \\
\hline & Antisense & 5'-GCGAGACATGATGGCATCT-3' \\
\hline \multirow[t]{2}{*}{ S100A8 } & Sense & 5'-ATGCCGTCTACAGGGATGAC-3' \\
\hline & Antisense & 5'-ACTGAGGACACTCGGTCTCT-3' \\
\hline \multirow[t]{2}{*}{ GAPDH } & Sense & 5'-ATGGGGAAGGTGAAGGTCG-3' \\
\hline & Antisense & 5'-GGGGTCATTGATGGCAACAATA-3' \\
\hline \multicolumn{3}{|l|}{ Primers for siRNA } \\
\hline \multirow[t]{2}{*}{ Si S100A8 001} & Sense & 5'-CUAUCAUCGACGUCUACCATT-3' \\
\hline & Antisense & 5'-UGGUAGACGUCGAUGAUAGTT-3' \\
\hline \multirow[t]{2}{*}{ Si S100A8 002} & Sense & 5'-GGAAUUUCCAUGCCGUCUATT-3' \\
\hline & Antisense & 5'-UAGACGGCAUGGAAAUUCCTT-3' \\
\hline \multirow[t]{2}{*}{ Si S100A8 003} & Sense & 5'-GACGUCUGGUUCAAAGAGUTT-3' \\
\hline & Antisense & 5'-ACUCUUUGAACCAGACGUCTT-3' \\
\hline \multicolumn{3}{|c|}{ Primers for CHIP-qPCR } \\
\hline \multirow[t]{2}{*}{$-835 \sim-618$} & Sense & 5'-AAGCAGGTGAGTGGTTGTTAGGG-3' \\
\hline & Antisense & 5'-ACAAACAGAACACTTCCGTCCC-3' \\
\hline \multirow[t]{2}{*}{$-639 \sim-428$} & Sense & 5'-CAACAGTTTGACAAATACGGTGC-3' \\
\hline & Antisense & 5'-CCTAACAACCACTCACCTGCTTT-3' \\
\hline \multirow[t]{2}{*}{$-401 \sim-188$} & Sense & 5'-GGTGGTCGGAAGGTAATAAGGC-3' \\
\hline & Antisense & 5'-CATTTCATTCCCCTCAGGCTTGT-3' \\
\hline
\end{tabular}

\section{Immunofluorescence}

Fifty percent confluent cells were cultured on glass coverslips in the wells of 24 -well plates for $24 \mathrm{~h}$. Then cells were washed, fixed, and permeabilized with $0.3 \%$ Triton $\mathrm{X}-100$ for $10 \mathrm{~min}$. After blocking with goat serum for 2 $\mathrm{h}$, cells were incubated for $1 \mathrm{~h}$ with antibody anti-S100A8 (1:30dillution) (Proteintech, Chicago, USA) and Anti-HRD1 (1:50 dilution) (Abcam, Cambridge, UK). Then, dishes were washed and incubated with Alexa Fluor 488 or Alexa Fluor 594-conjugated secondary antibodies (1:50 dilution) for $1 \mathrm{~h}$ at room temperature. Nuclei were stained with Hoechst (10 $\mathrm{mg} / \mathrm{ml}$ ) for $2 \mathrm{~min}$. Samples were examined with fluorescence microscope (Zeiss, Oberkochen, German).

\section{Animal tumor model}

Female athymic nude mice (6-weeks-old) were purchased from Shanghai Laboratory Animal Centre (Chinese Academy of Sciences, Shanghai, China) and maintained in cage housing under specific pathogen-free conditions. Cultured MCF7/Tam cells were transfected with EGFP or EGFP-tagged HRD1 lentivirus according to previous report [7]. Cells were injected either into the right or left flank region of the mice to generate subcutaneous xenografts. Tamoxifen was given once the size of the xenograft reached approximately $4 \mathrm{~mm}$ in diameter. The mice were randomly assigned into two groups of eight. They were treated with Tamoxifen by intragastric administration with dosage of $25 \mathrm{mg} / \mathrm{kg}$ (twice a day) for 2 weeks. Tumor volumes were estimated using the formula: $0.5 \times$ length $\times$ width $^{2}$. This study was carried out in accordance with the guidelines of the Institutional Animal Care and Use Committee at Nanjing Medical University and was approved by the Committee on the Ethics of Animal Experiments of Nanjing Medical University.

\section{Statistical analysis}

Data were analyzed using the SPSS statistics 16.0 software package. Comparisons were performed using the Student's $t$ test between two groups or ANOVA in multiple groups. A $P$ value $<0.05$ was considered to be statistically significant.

\section{ACKNOWLEDGMENTS}

This work was supported by grants from: (1) the Emergency Management Program of National Natural Science Foundation of China (81541059) and the General Program of National Natural Science Foundation of China (81171363) to Wanhua Guo; (2) the General Program of Public Health Bureau of Nanjing (YKK15056) to Zhijun 
Jia. (3) Natural Science Foundation of Jiangsu Province (KZ03007140088) to Gareth Jenkins.

\section{CONFLICTS OF INTEREST}

The authors declare no conflict of interest.

\section{REFERENCES}

1. Shajahan-Haq AN, Cook KL, Schwartz-Roberts JL, Eltayeb AE, Demas DM, Warri AM, Facey CO, HilakiviClarke LA, Clarke R. MYC regulates the unfolded protein response and glucose and glutamine uptake in endocrine resistant breast cancer. Mol Cancer. 2014; 13:239.

2. Clarke R, Liu MC, Bouker KB, Gu Z, Lee RY, Zhu Y, Skaar TC, Gomez B, O'Brien K, Wang Y, HilakiviClarke LA. Antiestrogen resistance in breast cancer and the role of estrogen receptor signaling. Oncogene. 2003; 22:7316-7339.

3. Bao YI, Wang A, Mo J. S100A8/A9 is associated with estrogen receptor loss in breast cancer. Oncol Lett. 2016; 11:1936-1942.

4. Yang M, Zeng P, Kang R, Yu Y, Yang L, Tang D, Cao L. S100A8 contributes to drug resistance by promoting autophagy in leukemia cells. PloS one. 2014; 9:e97242.

5. Hsu YL, Hung JY, Tsai EM, Wu CY, Ho YW, Jian SF, Yen MC, Chang WA, Hou MF, Kuo PL. Benzyl butyl phthalate increases the chemoresistance to doxorubicin/ cyclophosphamide by increasing breast cancer-associated dendritic cell-derived CXCL1/GROalpha and S100A8/A9. Oncol Lett. 2015; 34:2889-2900.

6. Acharyya S, Oskarsson T, Vanharanta S, Malladi S, Kim J, Morris PG, Manova-Todorova K, Leversha M, Hogg N, Seshan VE, Norton L, Brogi E, Massague J. A CXCL1 paracrine network links cancer chemoresistance and metastasis. Cell. 2012; 150:165-178.

7. Xu YM, Wang HJ, Chen F, Guo WH, Wang YY, Li HY, Tang JH, Ding Y, Shen YC, Li M, Xuan WY, Liu LH, Wang J, et al. HRD1 suppresses the growth and metastasis of breast cancer cells by promoting IGF-1R degradation. Oncotarget. 2015; 6:42854-42867. doi: 10.18632/oncotarget.5733.

8. Nandakumar V, Vaid M, Katiyar SK. (-)-Epigallocatechin3-gallate reactivates silenced tumor suppressor genes, Cip1/ p21 and p16INK4a, by reducing DNA methylation and increasing histones acetylation in human skin cancer cells. Carcinogenesis. 2011; 32:537-544.

9. Tseng TH, Chien MH, Lin WL, Wen YC, Chow JM, Chen CK, Kuo TC, Lee WJ. Inhibition of MDA-MB-231 breast cancer cell proliferation and tumor growth by apigenin through induction of $\mathrm{G} 2 / \mathrm{M}$ arrest and histone $\mathrm{H} 3$ acetylation-mediated p21 expression. Environ Toxicol. 2016.

10. Nilsson U, Johnsson R, Fransson LA, Ellervik U, Mani K. Attenuation of tumor growth by formation of antiproliferative glycosaminoglycans correlates with low acetylation of histone H3. Environ Toxicol. 2010; 70:3771-3779.

11. Wort SJ, Ito M, Chou PC, Mc Master SK, Badiger R, Jazrawi E, de Souza P, Evans TW, Mitchell JA, Pinhu L, Ito K, Adcock IM. Synergistic induction of endothelin-1 by tumor necrosis factor alpha and interferon gamma is due to enhanced NF-kappaB binding and histone acetylation at specific kappaB sites. J Biol Chem. 2009; 284:24297-24305.

12. Chou SD, Khan AN, Magner WJ, Tomasi TB. Histone acetylation regulates the cell type specific CIITA promoters, MHC class II expression and antigen presentation in tumor cells. Int Immunol. 2005; 17:1483-1494.

13. Jaenisch R, Bird A. Epigenetic regulation of gene expression: how the genome integrates intrinsic and environmental signals. Nature genetics. 2003; 33:245-254.

14. Bird A. Perceptions of epigenetics. Nature. 2007; 447:396-398.

15. Yu SL, Lee DC, Son JW, Park CG, Lee HY, Kang J. Histone deacetylase 4 mediates SMAD family member 4 deacetylation and induces 5-fluorouracil resistance in breast cancer cells. Oncol Rep. 2013; 30:1293-1300.

16. Giessrigl B, Schmidt WM, Kalipciyan M, Jeitler M, Bilban M, Gollinger M, Krieger S, Jager W, Mader RM, Krupitza G. Fulvestrant induces resistance by modulating GPER and CDK6 expression: implication of methyltransferases, deacetylases and the hSWI/SNF chromatin remodelling complex. Brit J Cancer. 2013; 109:2751-2762.

17. Tsuchimochi K, Yagishita N, Yamasaki S, Amano T, Kato Y, Kawahara K, Aratani S, Fujita H, Ji F, Sugiura A, Izumi T, Sugamiya A, Maruyama I, et al. Identification of a crucial site for synoviolin expression. Mol Cell Biol. 2005; 25:7344-56.

18. Kaneko M, Yasui S, Niinuma Y, Arai K, Omura T, Okuma Y, Nomura Y. A different pathway in the endoplasmic reticulum stress-induced expression of human HRD1 and SEL1 genes. FEBS Lett. 2007; 581:5355-60.

19. Huang Y, Yang X, Xu T, Kong Q, Zhang Y, Shen Y, Wei Y, Wang G, Chang KJ. Overcoming resistance to TRAILinduced apoptosis in solid tumor cells by simultaneously targeting death receptors, c-FLIP and IAPs. Int J Oncol. 2016; 49:153-163. 